Document downloaded from:

http://hdl.handle.net/10251/50783

This paper must be cited as:

Rubio Arjona, L.; Reig, J.; Rubio Arjona, L. (2010). Analytical Approach to Model the Fade Depth and the Fade Margin in UWB Channels. IEEE Transactions on Vehicular Technology. 59(9):4214-4221. doi:10.1109/TVT.2010.2070815.

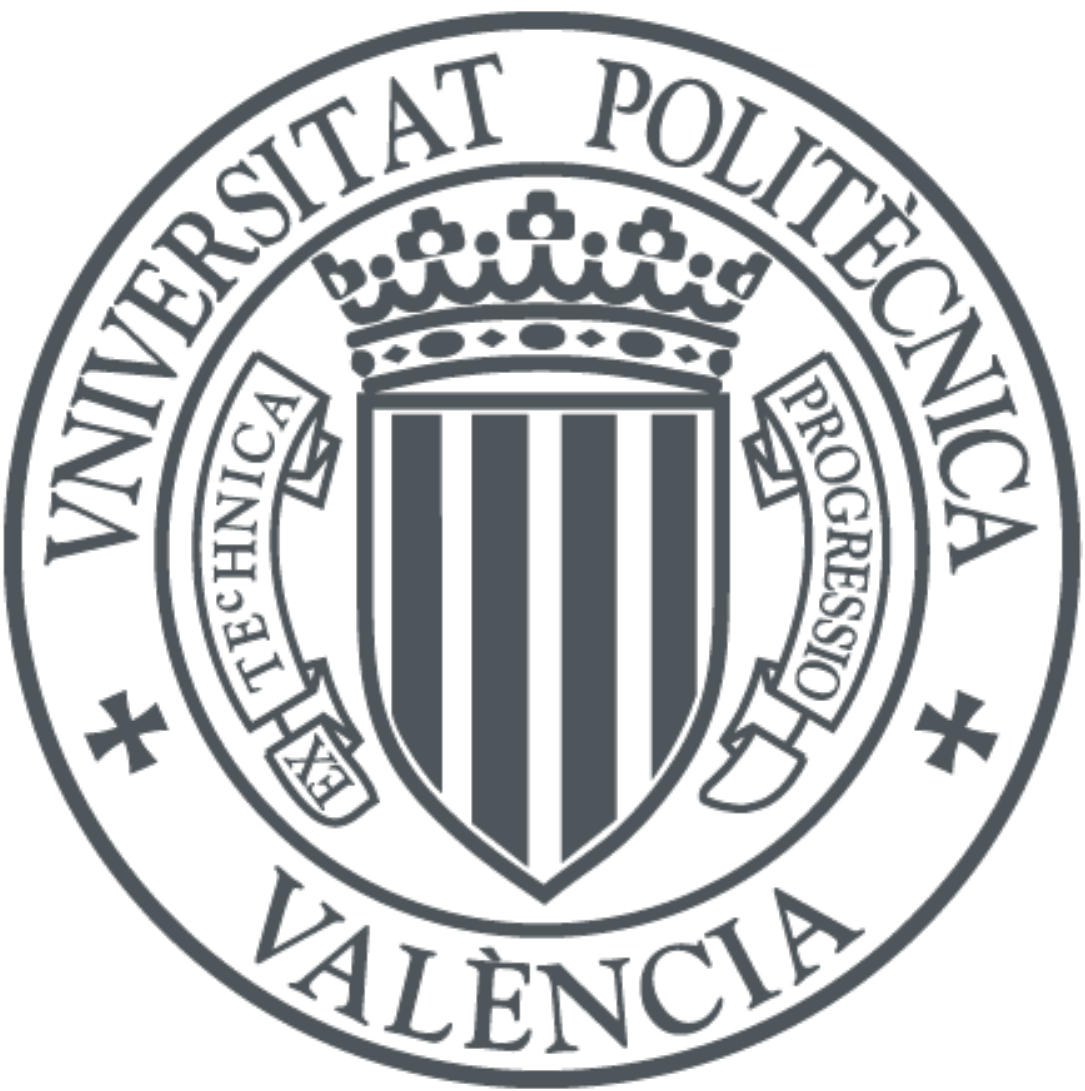

The final publication is available at

http://dx.doi.org/10.1109/TVT.2010.2070815

Copyright Institute of Electrical and Electronics Engineers (IEEE)

Additional Information 


\section{Analytical approach to model the fade depth and the fade margin in UWB channels}

Gonzalo Llano, Juan Reig ${ }^{1}$, Member IEEE, and Lorenzo Rubio-Arjona, Member IEEE Radio and Wireless Communication Group (RWCG). iTEAM Research Institute, Universidad Politécnica de Valencia

e-mail: gonllara@doctor.upv.es, jreigp@dcom.upv.es, lrubio@dcom.upv.es.

\footnotetext{
${ }^{1}$ Correspondence Address: Dr. Juan Reig, jreigp@dcom.upv.es, phone: +34 96 3879762, fax: +34 963877309. Radio and Wireless Communication Group (RWCG). iTEAM Research Institute, Universidad Politécnica de Valencia, 46022 Valencia (Spain).
} 


\title{
Analytical approach to model the fade depth and the fade margin in UWB channels
}

\begin{abstract}
In this letter, the variations of the ultra-wideband (UWB) channel power as a function of the channel bandwidth are investigated. An analytical approach to characterize the fade depth and the fade margin due to small-scale fading in indoor environments is proposed. The approach is based on the IEEE 802.15.4a ultra-wideband channel model and the assumption that the channel power can be modeled by a Gamma distribution. This analytical approach is checked by comparison with results derived through Monte Carlo simulations. The results show that the fade depth and the fade margin are closely related to the channel bandwidth. This analytical approach can be used to design and implement UWB communications systems.
\end{abstract}

Keywords- fade depth, fade margin, Nakagami-m fading, UWB channel power.

\section{INTRODUCTION}

Over recent years, ultra-wideband (UWB) communications systems have generated much interest as a consequence of the Federal Communications Commission (FCC) assent the $3.1 \mathrm{GHz}$ to $10.6 \mathrm{GHz}$ frequency range for commercial applications in 2002 [1]. Multipath fading resistance and high data rate transmission capacity, being the main characteristics of the UWB technology [2], render such UWB technology an excellent candidate for many indoor and short-range applications as compared to other wireless technologies. Applications of UWB can be found in high data rate wireless personal area networks (HDR-WPAN), positioning, location and home network communications related to multimedia applications [3].

The development of UWB communications systems requires a proper channel power characterization related to the propagation environment. Given the wideband nature of the UWB signal (a bandwidth of $7.5 \mathrm{GHz}$ ), it is of paramount importance to characterize the channel power variations in terms of the channel bandwidth in order to evaluate the performance of UWB applications. 
It is well known that in wireless channels the multipath propagation causes destructive signal interference leading to small-scale fading. In an unresolved multipath components (MPCs) channel, the received signal can suffer severe fading increasing the system outage probability and degrading its performance [4]. In view of the fact that multipath propagation can produce received signal fade, it is necessary to provide additional power in the link budget to enhance the system quality. This additional power is known as fade margin [5]. Other parameter to understand the small-scale fading concept is the fade depth that is referred to the received signal power variations about its local mean [6].

In order to have a complete description of the link budget and to define accurately the receiver sensitivity, a proper characterization of the channel power behavior is necessary. In this sense, the fade depth, the fade margin and the average power are important parameters to obtain an adequate description of the link budget, because they condition the final outage probability, and their knowledge is very useful to the radio network planning [4]. It is well known that the fade depth and the fade margin depend on the channel bandwidth, the transmitted-received distance [7], and the small-scale fading conditions. Therefore, their dependence is closely related to the environment where the propagation occurs.

Due to the importance of these parameters in the radio network planning, they have been extensively analyzed in the literature, especially in narrowband channels. In [5], the fade depth and fade margin are evaluated for a Rician channel as a function of the equivalent received bandwidth, showing that the fade margin variation is related to the channel bandwidth and that it falls monotonically when the channel bandwidth increases. In [6], the dependence of the received signal level distribution on the channel bandwidth is studied by computer simulations, showing that the fade depth has a strong dependence on the equivalent channel bandwidth. In [8], a relationship between the fade depth and the channel bandwidth is derived from a measurements campaign carried out in an indoor scenario. Therefore, the study of the average signal level, the fade depth and the fade margin in wideband transmission systems is a key issue for the development of wireless systems.

Since UWB systems employ a bandwidth higher than $500 \mathrm{MHz}[1]$, an adequate characterization of the channel power variations is necessary to deploy such systems. In this letter, we propose as a novel contribution an analytical approach to derive the 
fade depth and fade margin under the assumption that the received power is Gamma distributed. In our investigation, we have considered the IEEE 802.15.4a UWB channel model developed for indoor and outdoor environments in low data rate WPAN applications [9], where the wireless channel is assumed quasi-static during the symbol transmission [10]. We have checked the results derived from the analytical approach with Monte Carlo simulation results for several environments described in the UWB IEEE 802.15.4a channel model.

The letter is organized as follows: an introduction to the IEEE 802.15.4a UWB channel model is described in Section II. An analytical approach for the evaluation of the fade depth and the fade margin is investigated for different environments in Section III. Finally, conclusions are given in Section IV.

\section{IEEE 802.15.4a UWB CHANNEL MODEL}

In our analysis, we have considered the UWB channel model specified by the IEEE 802.15.4a Task Group (TG4a) [9]. This channel model is a modified version of the Saleh-Valenzuela (SV) model for indoor environments [11]. The channel impulse response (CIR) of the discrete multipath channel, denoted by $h(\tau)$, is expressed in agreement with [9] as

$$
h(\tau)=\sum_{l=1}^{L_{c}} \sum_{k=1}^{L_{r}} \alpha_{k, l} e^{j \varphi_{k, l}} \delta\left(\tau-T_{l}-\tau_{k, l}\right),
$$

where $l$ and $k$ represent the cluster and ray indexes within the $l$ th cluster, respectively; $\alpha_{k, l}$ and $\varphi_{k, l}$ correspond to the multipath gain coefficient and phases of the $k$ th ray in the $l$ th cluster, respectively; $T_{l}$ is the arrival time of the $l$ th cluster; and $\tau_{k, l}$ is the arrival time (in relation to $T_{l}$ ) of the $k$ th ray in the $l$ th cluster.

The cluster arrival time and the ray arrival time within each cluster are modeled as a Poisson distribution with arrival rates $\Lambda$ and $\lambda$, respectively, with $\lambda>\Lambda$. The MPCs amplitudes, $\alpha_{k, l}$, follow a Nakagami- $m$ distribution and they are mutually independent random variables (RVs). The phase terms $\varphi_{k, l}$ are uniformly distributed between 0 and $2 \pi$.

In the channel model, the number of clusters, $L_{c}$, is a Poisson distributed RV with probability density function (PDF) given by [9]

$$
p_{L_{c}}\left(L_{c}\right)=\frac{\overline{\left(L_{c}\right)}}{L_{c} !} \exp \left(-\overline{L_{c}}\right),
$$


where $\overline{L_{c}}$ is the mean number of clusters. According to this model, the statistics of the cluster inter-arrival times are described by a negative exponential RV whose PDF can be written as $[9]$

$$
p_{T}\left(T_{l} \mid T_{l-1}\right)=\Lambda \exp \left[-\Lambda\left(T_{l}-T_{l-1}\right)\right], l>0 .
$$

Due to the discrepancy in the fitting for indoor residential, and indoor and outdoor office environments [9], the TG4a proposes to model the ray arrival times with mixtures of two Poisson processes, as follows

$$
p_{\tau}\left(\tau_{k, l} \mid \tau_{(k-1), l}\right)=\beta \lambda_{1} \exp \left[-\lambda_{1}\left(\tau_{k, l}-\tau_{(k-1), l}\right)\right]+(1-\beta) \lambda_{2} \exp \left[-\lambda_{2}\left(\tau_{k, l}-\tau_{(k-1), l}\right)\right],
$$

where $\beta$ is the mixture probability, and $\lambda_{1}$ and $\lambda_{2}$ are the ray arrival rates of both Poisson processes. Then, the power delay profile (PDP) is exponentially distributed within each cluster and the power of each MPC can be calculated as

$$
\Omega_{k, l}=E\left[\alpha_{k, l}^{2}\right]=\Omega_{l} \frac{1}{\gamma_{l}\left[(1-\beta) \lambda_{1}+\beta \lambda_{2}+1\right]} \exp \left(-\tau_{k, l} / \gamma_{l}\right),
$$

where $\Omega_{l}$ is the mean power of the $l$ th cluster, $E[\cdot]$ denotes expectation, and $\gamma_{l}$ is the intra-cluster decay time constant. The mean power of the lth cluster, denoted by $\Omega_{l}$, follows an exponential decay, and in agreement with [9], $\Omega_{l}$ can be calculated as

$$
10 \log \left(\Omega_{l}\right)=10 \log \left[\exp \left(-T_{l} / \eta\right)\right]+M_{\text {cluster }}
$$

where $M_{\text {cluster }}$ is a Gaussian distributed RV with standard deviation $\sigma_{\text {cluster }}$. The parameter $\gamma_{l}$, depends linearly on the cluster inter-arrival time and it can be expressed as $\gamma_{l}=k T_{l}+\gamma_{0}$, being $k$ and $\gamma_{0}$ the parameters of the model. The channel multipath gain amplitudes, $\alpha_{k, l}$, are modeled as a Nakagami- $m$ distribution with PDF given by $[12]$

$$
p_{a_{k, l}}\left(a_{k, l}\right)=\frac{2}{\Gamma\left(m_{k, l}\right)}\left(\frac{m_{k, l}}{\Omega_{k, l}}\right)^{m_{k, l}} \quad a_{k, l}^{2 m_{k, l}-1} \exp \left(-\frac{m_{k, l}}{\Omega_{k, l}} a_{k, l}^{2}\right), \quad m_{k, l} \geq 0.5,
$$

where $m_{k, l}$ is the fading parameter of the $k$ th path inside the $l$ th cluster; $\Gamma(\cdot)$ is the Gamma function; and $\Omega_{k, l}$ is the mean power of the $k$ th path within the $l$ th cluster given by (5). The $m_{k, l}$ parameter is modeled as a lognormal distributed RV, whose logarithm has a mean $\mu_{m}$ and standard deviation $\sigma_{m}$, given by [9]

$$
\mu_{m}(\tau)=m_{0}-k_{m}(\tau)
$$




$$
\sigma_{m}(\tau)=\hat{m}_{0}-\hat{k}_{m}(\tau)
$$

where $m_{0}, k_{m}, \hat{m}_{0}$ and $\hat{k}_{m}$ are parameters of the model. The CIR of the UWB channel can be related to its channel transfer function (CTF) through the Fourier transform (FT). The FT of the CIR given by (1), and denoted by $H(f)$, can be calculated as

$$
H(f)=\sum_{l=1}^{L_{c}} \sum_{k=1}^{L_{r}} \alpha_{k, l} \exp \left[-j\left(2 \pi f\left(T_{l}+\tau_{k, l}\right)-\varphi_{k, l}\right)\right] .
$$

From the Parseval relation [13], the UWB channel power inside the channel bandwidth $\Delta f=f_{2}-f_{1}$, denoted by $\Psi_{\Delta f}$, is calculated in the frequency domain as

$$
\Psi_{\Delta f}=\int_{f_{1}}^{f_{2}}|H(f)|^{2} d f
$$

where $|H(f)|$ is the magnitude of the CTF, and $f_{1}$ and $f_{2}$ are the lower and upper frequencies, respectively.

\section{Analytical Approach of the Power Distribution, the Fade Depth And THE FADE MARGIN}

In this section, we propose an analytical approach to evaluate the power distribution, the fade depth and the fade margin as a function of the channel bandwidth. This approach is based on the IEEE 802.15.4a channel model described previously. Asymptotic values for the fade depth and the fade margin are derived and compared with simulation results for indoor residential and outdoor environments in both line-of-sight (LOS) and non-line-of-sight (NLOS) conditions.

Simulation results have been performed using the Monte Carlo (MC) method. For each environment considered, 1000 realizations of a small local area have been simulated, modeling the number of clusters, rays, cluster arrival and ray arrival times. The small local area corresponds to a small region around the receiver, in which the number of clusters and rays are constant, and only the phase and amplitude of rays change for short displacements. Then, for each realization, 60000 simulations of the MPCs phase and amplitude have been performed to model the power channel variations.

A. Channel power: We have assumed total independence between a pair of MPCs amplitude coefficients, in accordance with [14], [15], where the correlation coefficients between the amplitude of two MPCs measured remains below 0.2 [14], and 0.35 [15]. 
After some mathematical operations in (11), the UWB channel power inside the bandwidth $\Delta f(\mathrm{~Hz})$, can be expressed as

$$
\begin{aligned}
\Psi_{\Delta f} & =\Delta f \sum_{l=1}^{L_{c}} \sum_{k=1}^{L_{r}} \alpha_{k, l}^{2} \\
& +\frac{1}{2 \pi} \sum_{l=1}^{L_{c}} \sum_{\substack{n=1 \\
\{l, k\} \neq\{n, m\}}}^{L_{c}} \sum_{\substack{L_{r} \\
\{}}^{L_{r}} \frac{\alpha_{k, l} \alpha_{m, n}}{\left(T_{l}+\tau_{k, l}\right)-\left(T_{n}+\tau_{m, n}\right)}\left(\sin \left(2 \pi f_{2} B_{l, n}^{k, m}\right)-\sin \left(2 \pi f_{1} B_{l, n}^{k, m}\right)\right)^{\prime}
\end{aligned}
$$

where $B_{l, n}^{k, m}=\left(\left(T_{l}+\tau_{k, l}\right)-\left(T_{n}+\tau_{m, n}\right)\right)+\left(\varphi_{k, l}-\varphi_{m, n}\right)$ and $\{l, k\} \neq\{n, m\}$ represents the condition of summation, $l \neq n$ OR $k \neq m$.

A comparison of the channel power PDF between simulated data and the Gamma approximation calculated using (12) for an indoor residential environment is shown in Fig. 1. The PDFs curves plotted correspond to a single realization (one small local area) of the indoor residential environment with LOS condition for different channel bandwidths $(\Delta f=2,5$, and $7 \mathrm{GHz})$. Other results that support the assumption that the Gamma distribution can be able to provide a good approximation to the channel power variations are shown in Fig. 2, where indoor residential and outdoor environments are considered in both LOS and NLOS conditions with a channel bandwidth equal to $1 \mathrm{GHz}$. It is worth to note that the results show a higher fading parameter in LOS compared to the NLOS condition for the same channel bandwidth and environment. The parameters used in the simulation results are summarized in Table I. The goodness-of-fit of the Gamma distribution to the simulated data in Fig. 1 and 2 has been assessed through the Kolmogorov-Smirnov (KS) test for a $5 \%$ significant degree [17].

The mean channel power for a channel bandwidth $\Delta f$ expressed in linear units can be calculated from (12) as

$$
\Omega_{\Delta f}=E\left[\Psi_{\Delta f}\right]=\Delta f \sum_{l=1}^{L_{c}} \sum_{k=1}^{L_{r}} E\left[\alpha_{k, l}^{2}\right]=\Delta f \sum_{l=1}^{L_{c}} \sum_{k=1}^{L_{r}} \Omega_{k, l},
$$

where $\Omega_{k, l}$ is the average power of each contribution calculated from (5). Now, we investigate the channel power dependence on the channel bandwidth, deriving an analytical expression for the fade depth and the fade margin.

B. Fade depth: The fade depth, denoted by $F_{n \sigma}$, can be defined as a measure of the channel power variation due to the small-scale fading [8]. In a statistically sense, the 
fade depth can be calculated as $n$ times, $n=1,2,3, \ldots$, the standard deviation, $\sigma$, of the channel power variations expressed in logarithmic units. Due to each MPC amplitude, $\alpha_{k, l}$, is modeled as a Nakagami- $m$ random variable and in accordance with the results show in Fig. 1 and 2, we have assumed that in a small local area around the receiver the channel power given by (12) can be modeled as a Gamma distribution. Under this assumption, the variance and the standard deviation of the channel power expressed in logarithmic units, $\Phi_{\Delta f}(\mathrm{dBm})=10 \log \left(\Psi_{\Delta f}(\mathrm{~mW})\right)$, are given, respectively, by [12]

$$
\begin{aligned}
& \operatorname{var}_{\Phi}=\left[\frac{10}{\ln (10)}\right]^{2} \psi^{\prime}\left(m_{\Delta f}\right), \\
& \sigma_{\Phi}=\frac{10}{\ln (10)} \sqrt{\psi^{\prime}\left(m_{\Delta f}\right)},
\end{aligned}
$$

where $\psi^{\prime}(a)=\frac{\partial^{2}}{\partial a^{2}}(\ln [\Gamma(a)])$ is the trigamma function [18,(6.4.1)] and $m_{\Delta_{f}}$ is the fading parameter of the channel power in a bandwidth $\Delta f$, defined as

$$
m_{\Delta f}=\frac{\left(E\left[\Psi_{\Delta f}\right]\right)^{2}}{E\left[\Psi_{\Delta f}^{2}\right]-\left(E\left[\Psi_{\Delta f}\right]\right)^{2}}
$$

Substituting (12) in (16) and after some mathematical operations, it yields

$$
m_{\Delta f}=\frac{\left(\Delta f \sum_{l=1}^{L_{c}} \sum_{k=1}^{L_{r}} \Omega_{k, l}\right)^{2}}{(\Delta f)^{2} \sum_{l=1}^{L_{c}} \sum_{k=1}^{L_{r}} \frac{\Omega_{k, l}^{2}}{m_{k, l}}+\frac{1}{2 \pi^{2}} \sum_{l=1}^{L_{c}} \sum_{\substack{n=1 \\\{l, k\} \neq\{n, m\}}}^{L_{c}} \sum_{\substack{L_{r} \\\left\{L_{r}\right.}}^{L_{m=1}} \frac{\Omega_{k, l} \Omega_{m, n}}{\left[\left(T_{l}+\tau_{k, l}\right)-\left(T_{n}+\tau_{m, n}\right)\right]^{2}} \times D_{l, n}^{k, m}},
$$

where $D_{l, n}^{k, m}=\sin ^{2}\left\{\pi\left[\left(T_{l}+\tau_{k, l}\right)-\left(T_{n}+\tau_{m, n}\right)\right] \Delta f\right\} ;\{l, k\} \neq\{n, m\}$ represents the condition of summation, $l \neq n$ OR $k \neq m$; and the $m_{k, l}$ parameter is calculated using (8) and (9). Thus, the fade depth can be analytically calculated and approximated using [19] as

$$
F_{n \sigma}=\frac{10 n}{\ln (10)} \sqrt{\psi^{\prime}\left(m_{\Delta f}\right)} \approx \frac{10 n}{\ln (10)} \sqrt{\frac{1}{m_{\Delta f}}+\frac{1}{2 m_{\Delta f}^{2}}+\frac{1}{6 m_{\Delta f}^{3}}} .
$$

The relative error of (18) series expansion is less than $6.6 \cdot 10^{-3}$ for $m_{\Delta f} \geq 1$, which corresponds to the values used in simulations. 
A comparison between the analytical approximation of the fade depth given by (18) and simulation results is shown in Fig. 3 for an indoor residential environment with NLOS condition. The channel parameters used in the simulation results are summarized in Table I. It can be observed that simulation and analytical results are very similar, which is in agreement with the assumption that the power in a channel bandwidth $\Delta f$ can be modeled by a Gamma distribution.

The results also show that in channel bandwidths less than $1 \mathrm{MHz}$, the fade depth $F_{n \sigma}$ is approximately constant $(5.5 \mathrm{~dB}$ for $n=1)$, as corresponds to the behavior of a narrowband channel without frequency diversity gain. From Fig. 3 we can also observe that $F_{n \sigma}$ converges asymptotically from approximately $2 \mathrm{GHz}(0.8 \mathrm{~dB}$ for $n=$ 1). The floor level of the fade depth is a consequence of the amplitude variations of the MPCs for short displacements of the receiver within a small local area. The maximum error between simulation and analytical results is approximately $0.45 \mathrm{~dB}$ for $n=1$, corresponding to $\Delta f=8 \mathrm{MHz}$.

We can find asymptotic expressions for the fading parameter of the Gamma distribution as a function of the delay spread of the UWB channel. Let $u=\pi\left[\left(T_{l}+\tau_{k, l}\right)-\left(T_{n}+\tau_{m, n}\right)\right] \Delta f$ be defined as the parameter of the approximation.

Case 1. Low values of $u$. In this case, $m_{\Delta f}$ is given by

$$
m_{\Delta f} \approx \frac{\left(\sum_{l=1}^{L_{c}} \sum_{k=1}^{L_{r}} \Omega_{k, l}\right)^{2}}{\sum_{l=1}^{L_{c}} \sum_{k=1}^{L_{r}} \frac{\Omega_{k, l}^{2}}{m_{k, l}}+\frac{1}{2} \sum_{l=1}^{L_{c}} \sum_{\substack{L_{c} \\\{l, k\} \neq\{n, m\}}}^{L_{k}} \sum_{k=1}^{L_{r}} \sum_{m=1}^{L_{r}} \Omega_{k, l} \Omega_{m, n}}
$$

In narrowband fading channels, it is possible to derive an asymptotic expression for the fade depth substituting $m_{\Delta f}$ given by (19) into (18). We can also obtain a high limit for a bandwidth $\Delta f$ as a function of the root mean square (rms) delay spread, denoted by $\sigma_{\tau}$. Using $m_{\Delta f}$ given by (19), the high limit of the channel bandwidth for the asymptotic value of the fade depth can be calculated from simulation results as

$$
\Delta f(\mathrm{GHz}) \approx \frac{0.106}{\sigma_{\tau}(\mathrm{ns})},
$$

with a relative error less than $5 \%$.

Case 2. High values of $u$. In this situation, $m_{\Delta f}$ can be approximated as 


$$
m_{\Delta f} \approx \frac{\left(\sum_{l=1}^{L_{c}} \sum_{k=1}^{L_{r}} \Omega_{k, l}\right)^{2}}{\sum_{l=1}^{L_{c}} \sum_{k=1}^{L_{r}} \frac{\Omega_{k, l}^{2}}{m_{k, l}}} .
$$

Note that (21) agrees with [12, (80)]. Then the fade depth can be calculated for high values of $u$ substituting $m_{\Delta f}$ given by (21) into (18). The low limit of the channel bandwidth for the asymptotic wideband fade depth can be calculated from simulation results as

$$
\Delta f(\mathrm{GHz}) \approx \frac{185}{\sigma_{\tau}(\mathrm{ns})}
$$

with a relative error less than $5 \%$.

C. Fade margin: The fade margin can be defined as the difference in channel power corresponding to a probability $P$ and the $50 \%$ of the cumulative distribution function $(\mathrm{CDF})$ of the received channel power. The fade margin associated to a probability $P$, denoted by $F M_{P}$, is related to the channel power by

$$
P=\operatorname{Pr}\left\{E\left[\Phi_{\Delta f}(\mathrm{dBm})\right]-F M_{P}(\mathrm{~dB}) \leq \Phi_{P}(\mathrm{dBm})\right\}
$$

being $\Phi_{\Delta f}(\mathrm{dBm})$ the channel power, expressed in $\mathrm{dBm}$, calculated from (12) in a channel bandwidth $\Delta f$, and $\Phi_{P}(\mathrm{dBm})$ the channel power not exceeded with a probability $P$. The mean channel power can be calculated using (A.1)-(A.5) of the Appendix as

$$
E\left[\Phi_{\Delta f}(\mathrm{dBm})\right]=\frac{10}{\ln (10)} \psi\left(m_{\Delta f}\right)-10 \log \left(\frac{m_{\Delta f}}{\Omega_{\Delta f}}\right),
$$

where $\psi(a)=\frac{\partial}{\partial a}(\ln [\Gamma(a)])$ is the digamma function $[18,(6.3 .1)]$. After some operations detailed in Section B of the Appendix, $\Phi_{P}(\mathrm{dBm})$ is given by

$$
\Phi_{P}(\mathrm{dBm})=10 \log \left(\frac{\Omega_{\Delta f}}{m_{\Delta f}}\right)+10 \log \left[Q^{-1}\left(m_{\Delta f}, 1-P\right)\right],
$$

where $Q^{-1}$ is the inverse of the regularized incomplete gamma function [20]. Substituting (24) and (25) in (23), we can obtain a closed form expression of the fade margin for the UWB channel as 


$$
F M_{P}(\mathrm{~dB})=\frac{10}{\ln (10)} \psi\left(m_{\Delta f}\right)-10 \log \left[Q^{-1}\left(m_{\Delta f}, 1-P\right)\right]
$$

where $m_{\Delta f}$ is given by (17). Note that the fade margin is independent of the mean channel power $\Omega_{\Delta f}$. For $P$ approximating to 0 , the regularized incomplete Gamma function $Q^{-1}$ can be asymptotically extended according to [21]. Therefore, the fade margin given by (26) can be written as

$$
\begin{aligned}
F M_{P}(\mathrm{~dB})_{P \rightarrow 0} \simeq & \frac{10 \psi\left(m_{\Delta f}\right)}{\ln (10)}-10 \log \left\{\omega+\frac{\omega^{2}}{\left(m_{\Delta f}+1\right)}+\frac{\left(3 m_{\Delta f}+5\right) \omega^{3}}{2\left(m_{\Delta f}+1\right)^{2}\left(m_{\Delta f}+2\right)},\right. \\
& \left.+\frac{\left[m_{\Delta f}\left(8 m_{\Delta f}+33\right)+31\right] \omega^{4}}{3\left(m_{\Delta f}+1\right)^{3}\left(m_{\Delta f}+2\right)\left(m_{\Delta f}+3\right)}+\cdots\right\}
\end{aligned}
$$

where $\omega=\left[\Gamma\left(m_{\Delta f}+1\right) P\right]^{\frac{1}{m_{\Delta f}}}$. We have found that the error using (27) increases as $m_{\Delta f}$. For a relative error between the closed form expression given by (26) and the approximation given by (27) equal to $1 \%$, and for 6 terms of the summation, the maximum value of $m_{\Delta f}$ is 9.9 for a probability $P=1 \%$ and 15.6 for a probability $P$ $=0.1 \%$.

Fig. 4 shows the fade margin $F M_{p}$ given by (26) for the indoor residential NLOS IEEE 802.15.4a channel model as a function of the channel bandwidth for three different probabilities: $P=5 \%, 10 \%$ and $20 \%$. Note that the fade margin in a channel bandwidth less than $1 \mathrm{MHz}$ is approximately constant, and the difference between the Gamma approximation and the simulation for these bandwidth values is around $0.05 \mathrm{~dB}$ for $P=20 \%, 0.05 \mathrm{~dB}$ for $P=10 \%$, and $0.25 \mathrm{~dB}$ for $P=5 \%$. Moreover, a maximum difference of $1 \mathrm{~dB}$ and $0.5 \mathrm{~dB}$ between the Gamma approximation and simulation results is found at $\Delta f=8 \mathrm{MHz}$, for $P=5 \%$ and $P=$ $10 \%$, respectively.

\section{CONCLUSIONS}

Within this letter we investigate the variations of the received power as a function of the bandwidth channel, taking the IEEE 802.15.4a channel model as our point of reference. The results show that the channel power can be modeled by a Gamma distribution. Under the assumption that the channel power is Gamma distributed, an analytical approach to characterize the fade depth and the fade margin for indoor 
and outdoor environments is proposed. Also, asymptotic expressions for the fading parameter of the Gamma distribution as a function of the channel rms delay spread are proposed and discussed. The performance of the analytical approach has been checked by comparison with simulation results considering different propagation conditions for indoor residential and outdoor environments. The results show that the fade depth is approximately constant for channel bandwidths below $1 \mathrm{MHz}$ (just about $5.5 \mathrm{~dB}$ for $n=1$ ), i.e, the fade depth is bandwidth independent for narrowband channels, and adopts an asymptotic convergence for channel bandwidths beyond 2 $\mathrm{GHz}$ (just about $0.8 \mathrm{~dB}$ for $n=1$ ). A similar behavior of the fade margin occurs in terms of the channel bandwidth. This analytical approach enables a proper evaluation of the link budget in terms of the bandwidth channel and it can be used to design and implement UWB communications systems.

\section{APPENDIX}

\section{A. Mean of a Gamma random variable in logarithmic units}

Let $\Psi_{\Delta f}$ be a Gamma $\mathrm{RV}$ with $\Omega_{\Delta f}=E\left[\Psi_{\Delta f}\right]$ and $m_{\Delta f}=\Omega_{\Delta f}^{2} / E\left[\left(\Psi_{\Delta f}-\Omega_{\Delta f}\right)^{2}\right]$. The $\mathrm{PDF}$ of $\Psi_{\Delta f}$ is given by

$$
f_{\Psi_{\Delta f}}\left(\Psi_{\Delta f}\right)=\frac{1}{\Gamma\left(m_{\Delta f}\right)}\left(\frac{m_{\Delta f}}{\Omega_{\Delta f}}\right)^{m_{\Delta f}} \Psi_{\Delta f}^{m_{\Delta f}-1} \exp \left(-\frac{m_{\Delta f} \Psi_{\Delta f}}{\Omega_{\Delta f}}\right), \Psi_{\Delta f} \geq 0, m_{\Delta f} \geq 0.5
$$

The PDF of $\Phi_{\Delta f}=10 \log \Psi_{\Delta f}$ can be calculated as

$$
f_{\Phi_{\Delta f}}\left(\Phi_{\Delta f}\right)=\frac{K}{\Gamma\left(m_{\Delta f}\right)}\left(\frac{m_{\Delta f}}{\Omega_{\Delta f}}\right)^{m_{\Delta f}} \exp \left(K m_{\Delta f} \Phi_{\Delta f}-\frac{m_{\Delta f}}{\Omega_{\Delta f}} e^{K \Phi_{\Delta f}}\right),-\infty<\Phi_{\Delta f}<\infty
$$

where $K=\ln (10) / 10$. The mean value of $\Phi_{\Delta f}$ is given by

$$
E\left[\Phi_{\Delta f}\right]=\frac{K}{\Gamma\left(m_{\Delta f}\right)}\left(\frac{m_{\Delta f}}{\Omega_{\Delta f}}\right)^{m_{\Delta f}} \int_{-\infty}^{\infty} \Phi_{\Delta f} \exp \left(K m_{\Delta f} \Phi_{\Delta f}-\frac{m_{\Delta f}}{\Omega_{\Delta f}} e^{K \Phi_{\Delta f}}\right) d \Phi_{\Delta f}
$$

Using the transformation, $u=\exp \left(K \Phi_{\Delta f}\right)$, we can evaluate the integral of (A.3) as

$$
E\left[\Phi_{\Delta f}\right]=\frac{1}{K \Gamma\left(m_{\Delta f}\right)}\left(\frac{m_{\Delta f}}{\Omega_{\Delta f}}\right)^{m_{\Delta f}} \int_{0}^{\infty} \ln (u) u^{m_{\Delta f}-1} \exp \left(-\frac{m_{\Delta f}}{\Omega_{\Delta f}} u\right) d u .
$$

We can solve integral (A.4) using [22, (4.352 1)] 


$$
\int_{0}^{\infty} x^{\nu-1} \exp (-\mu x) \ln (x) d x=\frac{\Gamma(\nu)}{\mu^{\nu}}[\psi(\nu)-\ln (\mu)] ; \operatorname{Re}(\mu)>0 \text { OR } \operatorname{Re}(\nu)>0 .
$$

Therefore, substituting $\nu=m_{\Delta f}$ and $\mu=m_{\Delta f} / \Omega_{\Delta f}$ in (A.5), the mean value of this Gamma RV distribution in logarithmic units can be expressed as (24).

\section{B. Percentile values of a Gamma distribution in logarithmic units}

The value not exceeded with a probability $P$ of a Gamma RV in logarithmic units, $\Phi_{P}$, whose $\operatorname{PDF}$ is given by (A.2), can be expressed as $P=\operatorname{Prob}\left\{\Phi_{\Delta f} \leq \Phi_{P}\right\}$. For convenience, we calculate this probability using the PDF of the corresponding Gamma distribution as

$$
\begin{aligned}
P & =\operatorname{Prob}\left\{\Phi_{\Delta f} \leq \Phi_{P}\right\}=\operatorname{Prob}\left\{\Psi_{\Delta f} \leq \Psi_{p}\right\}=\int_{0}^{\Psi_{p}} f_{\Psi_{\Delta f}}\left(\Psi_{\Delta f}\right) d \Psi_{\Delta f} \\
& =\frac{1}{\Gamma\left(m_{\Delta f}\right)}\left(\frac{m_{\Delta f}}{\Omega_{\Delta f}}\right)^{m_{\Delta f}} \int_{0}^{\Psi_{p}} \Psi_{\Delta f}^{m_{\Delta f}-1} \exp \left(-\frac{m_{\Delta f} \Psi_{\Delta f}}{\Omega_{\Delta f}}\right) d \Psi_{\Delta f} \\
& =\frac{1}{\Gamma\left(m_{\Delta f}\right)} \gamma\left(m_{\Delta f}, \frac{m_{\Delta f}}{\Omega_{\Delta f}} \Psi_{p}\right)
\end{aligned}
$$

where $\Psi_{\Delta f}$ is a Gamma RV whose PDF is given by (A.1), $\Psi_{P}=10^{\Phi_{P} / 10}$ and $\gamma(a, z)=\int_{0}^{z} e^{-t} t^{a-1} d t$ is the incomplete Gamma function [18, (6.5.2)]. If we define the regularized incomplete gamma function as $Q(a, z)=\Gamma(a, z) / \Gamma(a)$, where $\Gamma(a, z)=\Gamma(a)-\gamma(a, z)=\int_{z}^{\infty} e^{-t} t^{a-1} d t \quad[18, \quad(6.5 .3)]$, the inverse of the regularized incomplete Gamma function can be expressed as [20]

$$
s=Q(a, z) \rightarrow z=Q^{-1}(a, s) .
$$

According to (A.6) and substituting $a=m_{\Delta f}, z=m_{\Delta f} \Psi_{P} / \Omega_{\Delta f}$, and $s=1-P$ into (A.7), the value of $\Psi$ not exceeded with a probability $P, \Psi_{P}$, is given by

$$
\Psi_{P}=\frac{\Omega_{\Delta f}}{m_{\Delta f}} Q^{-1}\left(m_{\Delta f}, 1-P\right) .
$$

Consequently, the value of $\Phi_{P}$ not exceeded with a probability $P$ can be written as $(25)$. 


\section{REFERENCES}

[1] Federal Communications Commission: 'Revision of part 15 of the commission's rules regarding ultra-wideband transmission systems: first report and order'. Federal Communications Commission, USA, Washington, DC, USA, Tech. Rep. FCC 02-48, February 2002

[2] S. Roy, J. R. Foerster, V. S. Somayazulu, and D. G. Leeper, "Ultra-wideband radio design: the promise of high-speed, short-range wireless connectivity," Proc. IEEE, vol. 92, pp. 295-311, Feb. 2004.

[3] Y. Liuqing and G.B. Giannakis, "Ultra-wideband communications: an idea whose time has come," IEEE Signal Processing Mag., vol. 21, pp. 26-54, Nov. 2004 .

[4] W.C. Jakes, Microwave Mobile Communications. Wiley, New York, 1974.

[5] F. Cardoso and L. Correia, "Fading depth dependence on system bandwidth in mobile communications - an analytical approximation," IEEE Trans. Veh. Technol., vol. 52, pp. 587-593, May 2003.

[6] J. Yang and S. Kozono, "A study of received signal-level distribution in wideband transmissions in mobile communications," IEEE Trans. Veh. Technol., vol. 48, pp. 1718-1725, Sep. 1999.

[7] E.R. Bastidas-Puga, F. Ramírez-Mireles, and D. Muñoz-Rodríguez, "On fading margin in ultrawideband communications over multipath channels," IEEE Transactions on Broadcasting, vol. 51, pp. 366-370, Sep. 2005.

[8] W.Q. Malik, B. Allen, and D.J. Edwards, "Bandwidth dependent modeling of small scale fade depth in wireless channels," IET Microw. Antennas Propag., vol. 2, pp. 519-528, Sep. 2008.

[9] A.F. Molisch et al,. IEEE 802.15.4a channel model final report. Technical Report, Document IEEE 802.1504-0062-02-004a, 2005.

[10] H. Hashemi, "Impulse response modeling of indoor radio propagation channels," IEEE J. Select. Areas Commun., vol. 11, pp. 967-978, Sep. 1993.

[11] A.A. Saleh and R.A. Valenzuela, "A statistical model for indoor multipath propagation," IEEE J. Select. Areas Commun., vol. 5, pp. 128-137, Feb. 1987.

[12] M. Nakagami, "The $m$-distribution - A general formula of intensity distribution of rapid fading," in Statistical Methods in Radio Wave Propagation, W. C. Hoffman, Ed. Oxford, U.K.: Pergamon, 1960, pp. 3-36.

[13] J.G. Proakis, Digital Communications. Third Edition, McGraw-Hill Book Company, New York, 1995.

[14] D. Cassioli, M. Z. Win, and A.F. Molisch, "The ultra-wideband indoor channel: from statistical model to simulations," IEEE J. Select. Areas Commun., vol. 20, pp. 1247-1252, Aug. 2002. 
[15] C.-C. Chong and S. K. Yong, "A generic statistical-based UWB channel model for high-rise apartments," IEEE Trans. Antennas Propag., vol. 53, no. 8, pp. 2389-2399, Aug. 2005.

[16] F. Ramírez-Mireles, "Quantifying the degradation of combined MUI and multipath effects in impulse-radio UWB," IEEE Trans. Wireless Commun., vol. 6, pp. 2831-2836, Aug 2007.

[17] F. J. Massey, "The Kolmogorov-Smirnov test for goodness of fit," Journal of the American Statistical Association, vol. 46, no. 253, pp. 68-78, 1951.

[18] M. Abramowitz and I. A. Stegun, Handbook of Mathematical Functions; with Formulas, Graphs and Mathematical Tables. New York: Dover, 1972.

[19] WolframMathworld. (2009, Jan). [Online]. Available: http://functions.wolfram.com/06.15.06.0010.01. (Last access, 9/04/2009).

[20] WolframMathworld. (2009,Jan). [Online]. Available: http://functions.wolfram.com/06.12.02.0001.01. (Last access 9/04/2009).

[21] WolframMathworld. (2009,Jan). [Online]. Available: http://functions.wolfram.com/06.12.06.0007.01. (Last access 9/04/2009).

[22] I.S. Gradsthteyn and I.M. Ryzhik. Table of integrals, series and products. San Diego, CA: Academic, 5th ed., 1994. 


\section{FIGURE CAPTIONS}

Figure 1. Probability density function of the channel power, $\Psi_{\Delta f}$, in the indoor residential IEEE 802.15.4a UWB channel model with LOS condition and several values of channel bandwidth, $\Delta f=2,5$, and $7 \mathrm{GHz}$.

Figure 2. Probability density function of the channel power, $\Psi_{\Delta f}$, for indoor residential and outdoor environments with LOS and NLOS with a channel bandwidth $\Delta f=1 \mathrm{GHz}$.

Figure 3. Fade depth, $F_{n \sigma}$, for the indoor residential IEEE 802.15.4a UWB channel model derived from the analytical approach and simulation results in NLOS condition.

Figure 4. Fade margin, $F M_{P}$, in the indoor residential IEEE 802.15.4a UWB channel model derived from the analytical approach and simulation results in NLOS condition.

Table 1. IEEE 802.15.4a UWB channel model parameters. 
FIGURE 1

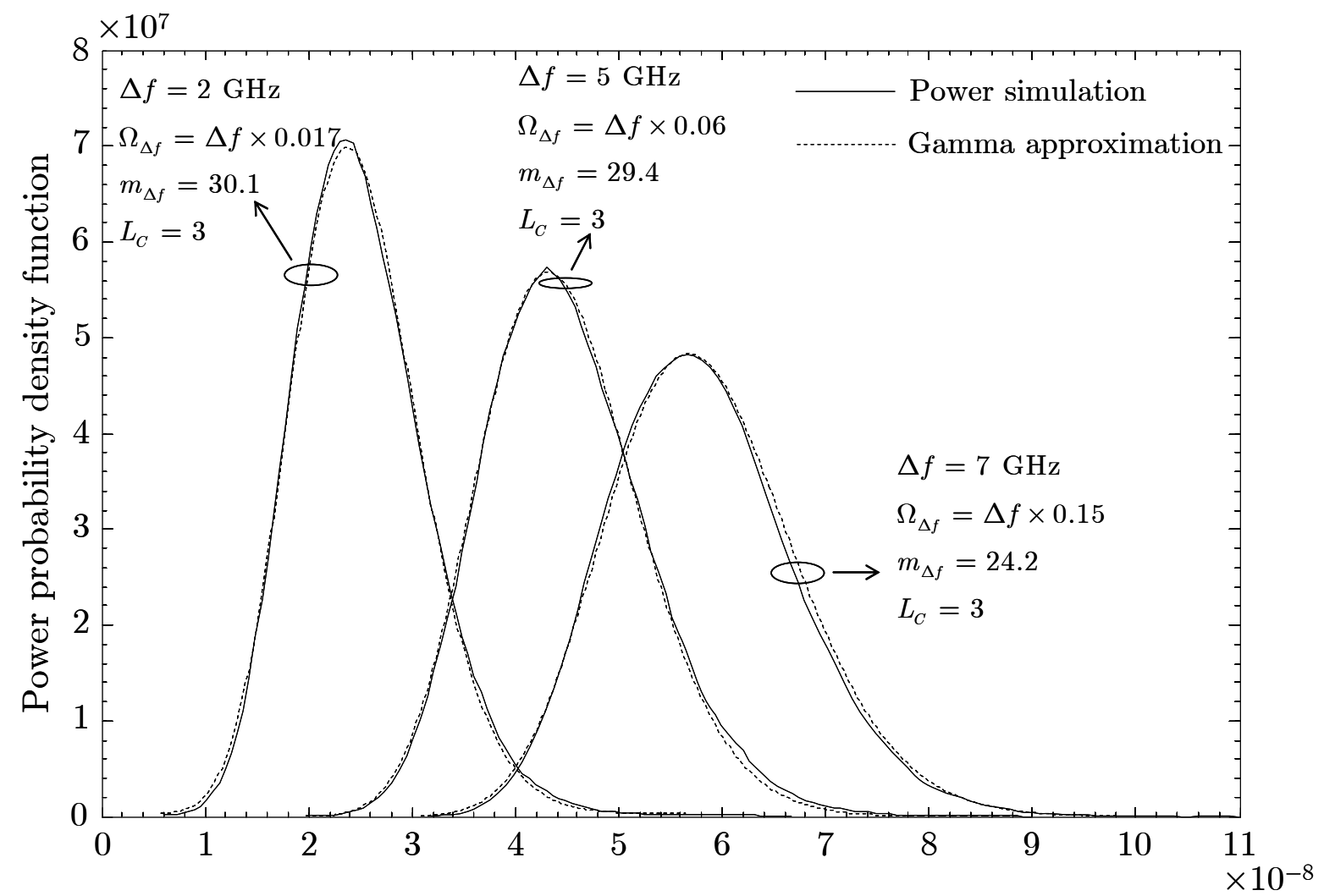


FIGURE 2

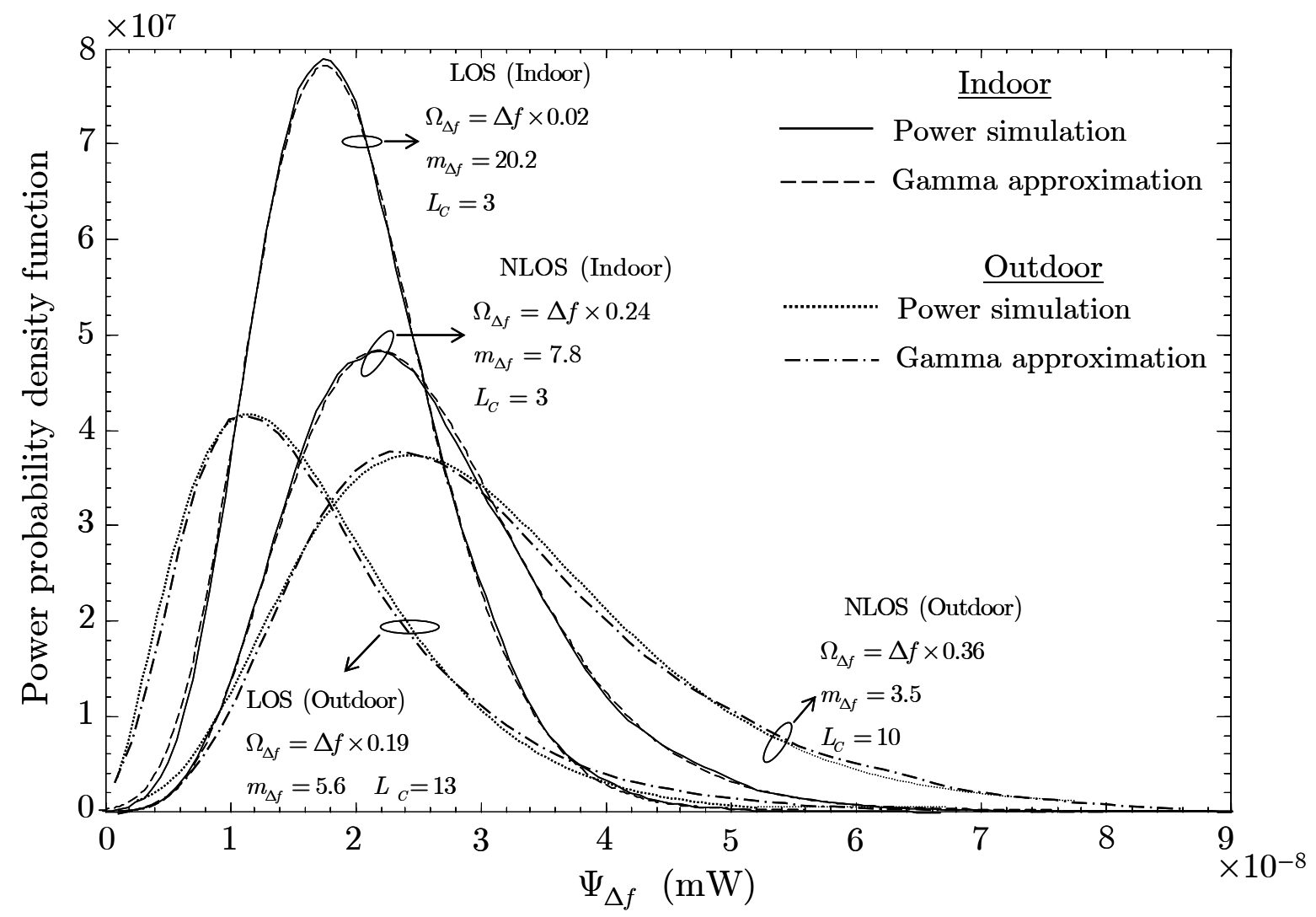


FIGURE 3

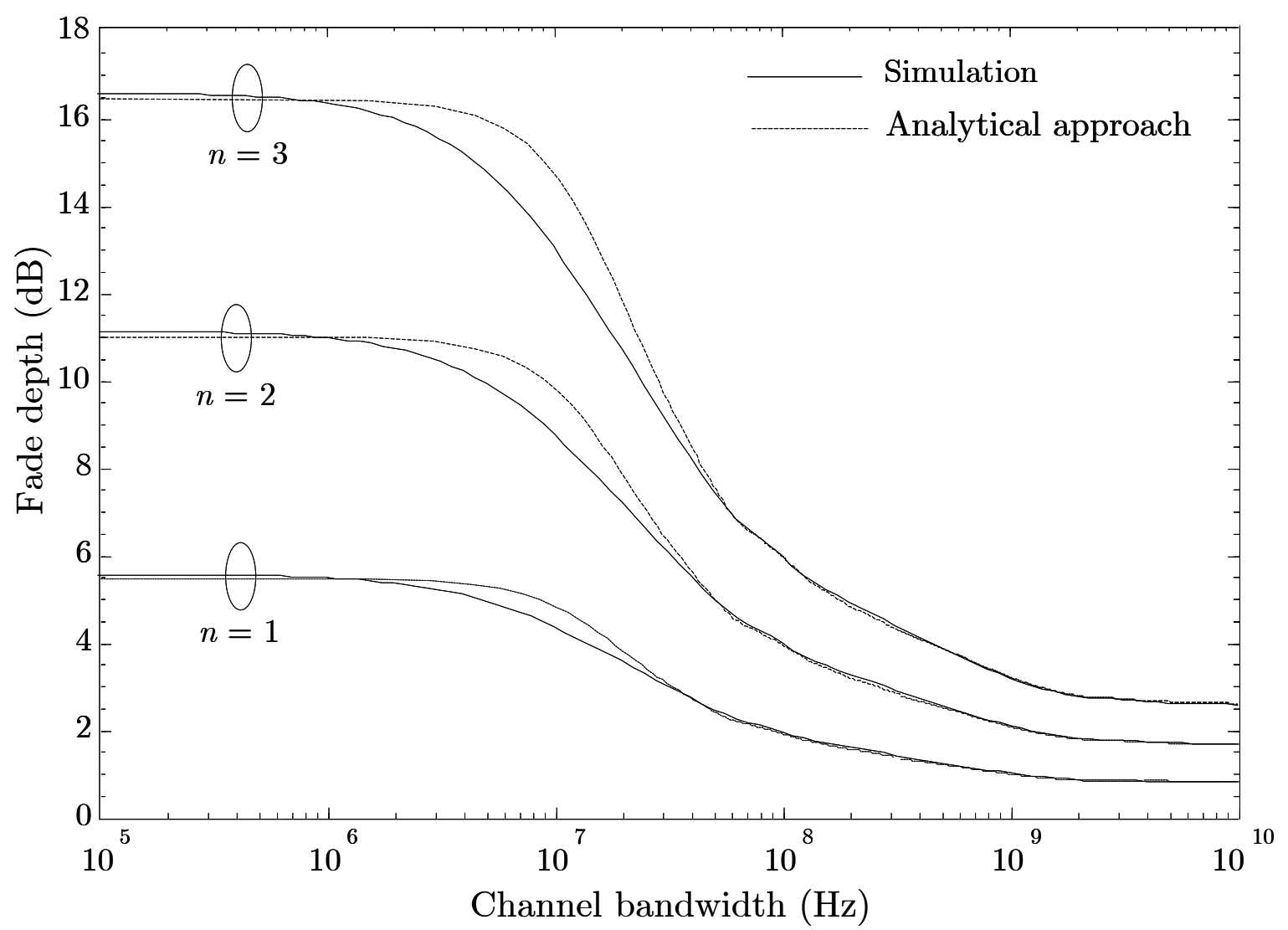


FIGURE 4

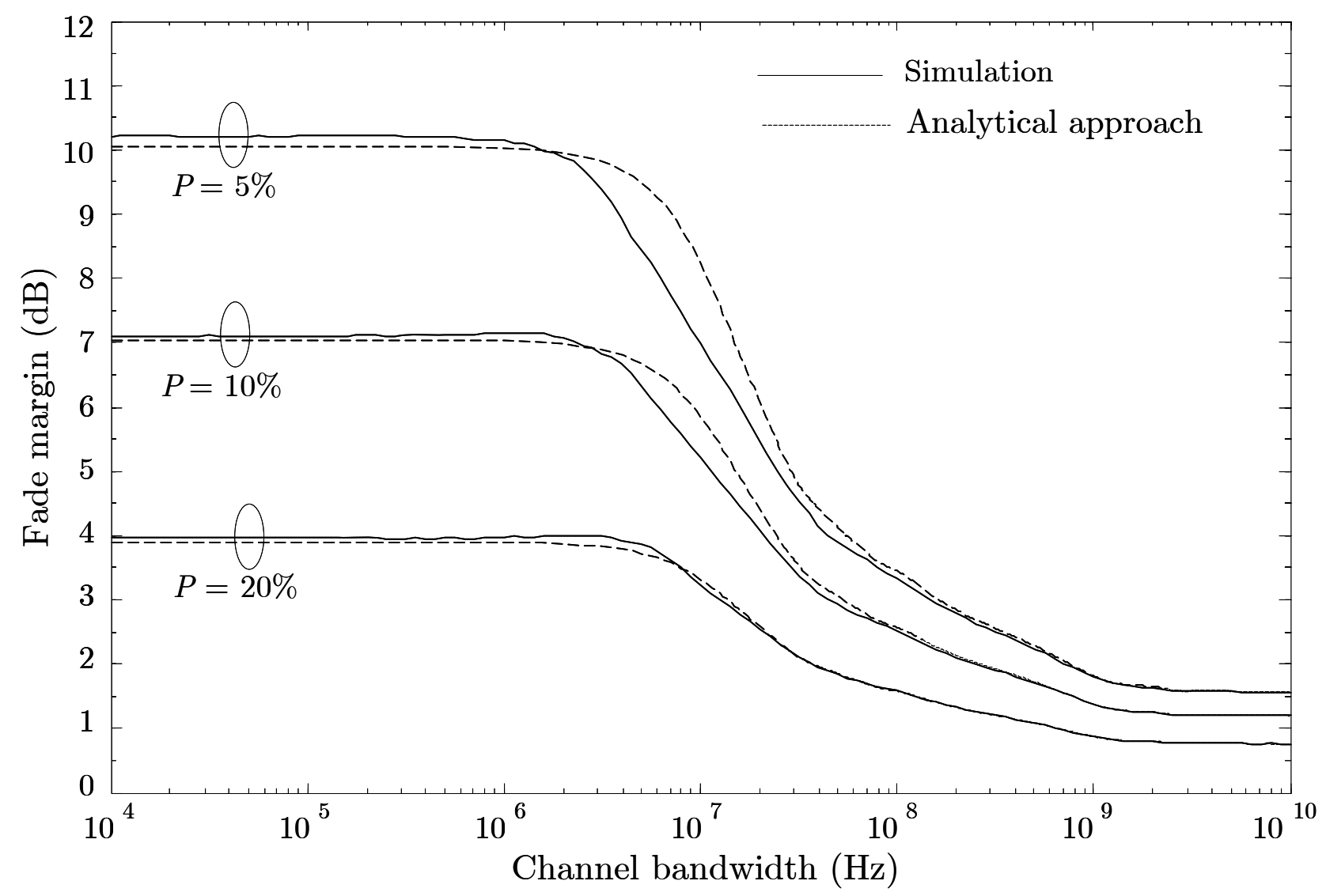


TABLE I

\begin{tabular}{|l|c|c|c|c|}
\hline Model parameters & \multicolumn{2}{|c|}{ Indoor residential } & \multicolumn{2}{c|}{ Outdoor } \\
\hline & LOS & NLOS & LOS & NLOS \\
\hline$\overline{L_{c}}$ & 3.0 & 3.5 & 13.6 & 10.5 \\
\hline$\Lambda(1 / \mathrm{ns})$ & 0.047 & 0.12 & 0.0048 & 0.0243 \\
\hline$\lambda_{1} / \lambda_{2}(1 / \mathrm{ns})$ & $1.54 / 0.15$ & $1.77 / 0.15$ & $0.13 / 2.41$ & $0.15 / 1.13$ \\
\hline$\beta$ & 0.095 & 0.045 & 0.0078 & 0.062 \\
\hline$\eta(\mathrm{ns})$ & 22.61 & 26.27 & 31.7 & 104.7 \\
\hline$\gamma_{0}(\mathrm{~ns})$ & 12.53 & 17.5 & 3.7 & 9.3 \\
\hline$\sigma_{\text {cluster }}(\mathrm{dB})$ & 2.75 & 2.93 & 3.0 & 3.0 \\
\hline$k_{\gamma}(\mathrm{d})$ & 0 & 0 & 0 \\
\hline$m_{0}(\mathrm{~dB})$ & 0.67 & 0.69 & 0.77 & 0.56 \\
\hline$k_{m}$ & 0 & 0 & 0 & 0 \\
\hline$\hat{m}_{0}(\mathrm{~dB})$ & 0.28 & 0.32 & 0,78 & 0,25 \\
\hline$\hat{k}_{m}$ & 0 & 0 & 0 & 0 \\
\hline
\end{tabular}

